\title{
A MODIFIED PERRY-TYPE DERIVATIVE-FREE PROJECTION METHOD FOR SOLVING LARGE-SCALE NONLINEAR MONOTONE EQUATIONS
}

\author{
M. Koorapetse@, P. Kaelo and S. Kooepile-Reikeletseng
}

\begin{abstract}
In this paper, a new modified Perry-type derivative-free projection method for solving largescale nonlinear monotone equations is presented. The method is developed by combining a modified Perry's conjugate gradient method with the hyperplane projection technique. Global convergence and numerical results of the proposed method are established. Preliminary numerical results show that the proposed method is promising and efficient compared to some existing methods in the literature.
\end{abstract}

Mathematics Subject Classification. 65K10, 90C06, 90C30, 65K05.

Received March 19, 2021. Accepted July 31, 2021.

\section{INTRODUCTION}

In this paper, we use a modified Perry-type derivative-free projection method to solve the nonlinear monotone equations

$$
F(x)=0, \quad x \in \Omega,
$$

where $F: \mathbb{R}^{n} \rightarrow \mathbb{R}^{n}$ is continuous and monotone, and $\Omega \subseteq \mathbb{R}^{n}$ is a nonempty closed convex set. By monotonicity, we mean that

$$
(F(x)-F(y))^{T}(x-y) \geq 0, \quad \forall x, y \in \mathbb{R}^{n} .
$$

If $\Omega=\mathbb{R}^{n}$ then (1.1) is a general system of nonlinear equations problem, and when $\Omega \subseteq \mathbb{R}^{n}$ is a nonempty closed convex set then (1.1) is said to be constrained. Nonlinear monotone equations have many practical applications such as in chemical equilibrium systems [20] and the economic equilibrium problems [9]. Also, some monotone variational inequality problems can also be converted into nonlinear monotone equations by means of fixed point mappings or normal maps if the underlying function satisfies some coercive conditions [34].

Conjugate gradient-based projection methods are among the most famous and efficient methods for solving (1.1) and, thus, have recently received a lot of attention. This is due to their simplicity, global convergence properties and low memory requirements, which make them suitable for solving large-scale equations $[8,16,26]$. They are iterative methods, that is, given $x_{k}$, the next iterate $x_{k+1}$ is obtained as

$$
x_{k+1}=P_{\Omega}\left[x_{k}-\frac{F\left(z_{k}\right)^{T}\left(x_{k}-z_{k}\right)}{\left\|F\left(z_{k}\right)\right\|^{2}} F\left(z_{k}\right)\right] \text {, }
$$

Keywords. Derivative-free, nonlinear monotone equations, Perry's conjugate gradient method, projection technique.

Department of Mathematics, University of Botswana, Private Bag UB00704, Gaborone, Botswana

* Corresponding author: koorapetsem@ub.ac.bw 
where $z_{k}=x_{k}+\alpha_{k} d_{k}, \alpha_{k}>0$ is a step length and

$$
d_{k}= \begin{cases}-F_{k}, & \text { if } k=0, \\ -F_{k}+\beta_{k} d_{k-1}, & \text { if } k \geq 1,\end{cases}
$$

with $\beta_{k}$ being a conjugate gradient parameter such that

$$
F_{k}^{T} d_{k} \leq-c\left\|F_{k}\right\|^{2}, \quad c>0
$$

where $F_{k}=F\left(x_{k}\right)$ and $\|\cdot\|$ is the Euclidean norm. If $\Omega$ is a closed convex subset of $\mathbb{R}^{n}$ then the projection operator $P_{\Omega}[\cdot]$ is a map from $\mathbb{R}^{n}$ onto $\Omega$, that is,

$$
P_{\Omega}[x]=\arg \min \{\|x-y\| \mid y \in \Omega\}, \quad \forall x \in \mathbb{R}^{n} .
$$

This operator is non-expansive, that is, for any $x, y \in \mathbb{R}^{n}$,

$$
\left\|P_{\Omega}[x]-P_{\Omega}[y]\right\| \leq\|x-y\| .
$$

Conjugate gradient-based projection methods are obtained by combining conjugate gradient methods $[3,5,6,30,32]$ with the projection technique proposed by Solodov and Svaiter [25]. Thus, they differ according to how the direction is obtained, or, more specifically, in how the parameter $\beta_{k}$ is constructed. Recently, by employing Perry's conjugate gradient parameter [22], in which

$$
\beta_{k}^{P}=\frac{F_{k}^{T}\left(y_{k-1}-s_{k-1}\right)}{y_{k-1}^{T} d_{k-1}}
$$

or the Dai-Liao conjugate gradient parameter [5], in which

$$
\beta_{k}^{\mathrm{DL}}=\frac{F_{k}^{T}\left(y_{k-1}-t s_{k-1}\right)}{y_{k-1}^{T} d_{k-1}}, t \geq 0,
$$

where $y_{k-1}=F_{k}-F_{k-1}$ and $s_{k-1}=x_{k}-x_{k-1}$, a number of modified forms of the Perry and Dai-Liao methods for nonlinear systems of equations have been proposed $[1,4,7,27,28]$. These Perry and Dai-Liao methods are based on a quasi-Newton aspect and have been considered to be among the most effective in the context of unconstrained optimization. Note that when $t=1$, the Dai-Liao method reduces to the Perry method.

In Dai et al. [7], a modified Perry method is combined with the hyperplane technique [25] to give a derivativefree method for solving large-scale nonlinear monotone equations. And in Waziri et al. [28], two enhanced DaiLiao methods are presented based on two modified spectral coefficients and a revised form of the extended secant condition in [29]. All these methods were shown to be very competitive when compared to some of the existing methods for solving large-scale nonlinear monotone equations.

Abubakar et al., in [2], constructed three-term conjugate gradient projection methods by proposing the direction as

$$
d_{k}= \begin{cases}-F_{k}, & \text { if } k=0, \\ -F_{k}+\beta_{k}^{C D} w_{k-1}-\lambda_{k} F_{k}, & \text { if } k \geq 1,\end{cases}
$$

with $\beta_{k}^{C D}=\frac{\left\|F_{k}\right\|^{2}}{-d_{k-1}^{T} F_{k}}$ and $w_{k-1}=z_{k-1}-x_{k-1}=\alpha_{k-1} d_{k-1}$. In order to satisfy (1.3), they derived the parameter $\lambda_{k}$ using three different approaches, thus proposing three different conjugate gradient projection methods with

$$
\lambda_{k}=\frac{F_{k}^{T} w_{k-1}}{-d_{k-1}^{T} F_{k}}, \quad \lambda_{k}=\frac{\left\|F_{k}\right\|^{2}\left\|w_{k-1}\right\|^{2}}{\left(-d_{k-1}^{T} F_{k}\right)^{2}} \quad \text { and } \quad \lambda_{k}=\frac{F_{k}^{T} w_{k-1}}{-d_{k-1}^{T} F_{k}}+\frac{\left\|F_{k}\right\|^{2}}{\left(-d_{k-1}^{T} F_{k}\right)^{2}},
$$

and they named the resulting algorithms as M3TCD1, M3TCD2 and M3TCD3, respectively. 
Another recently suggested three term derivative-free conjugate gradient-based projection method, which the authors named PDY, is that by Liu and Feng [16]. In this method, the authors proposed a three term derivative-free projection method based on the Dai-Yuan (DY) conjugate gradient parameter

$$
\beta_{k}=\frac{\left\|F_{k}\right\|^{2}}{d_{k-1}^{T} w_{k-1}},
$$

where

$$
y_{k-1}=F_{k}-F_{k-1}, w_{k-1}=y_{k-1}+t_{k-1} d_{k-1}, \text { and } t_{k-1}=1+\max \left\{0,-\frac{d_{k-1}^{T} y_{k-1}}{d_{k-1}^{T} d_{k-1}}\right\} .
$$

This method was shown to have nice convergence properties. And Yan et al., in [31], proposed two derivative-free projection methods based on the three term Hestenes-Stiefel (HS) conjugate gradient method of Zhang et al. [32]. They showed that their methods were also efficient for solving large-scale nonlinear systems of monotone equations. Based on a modified line search, an extension of the scaled conjugate gradient (SCG) method of [3] and the projection technique, Ou and $\mathrm{Li}$ [21] proposed a derivative-free SCG-type projection method for nonlinear monotone equations with convex constraints. They showed further that when $F$ in (1.1) is a strongly monotone mapping, then the sequence $\left\{x_{k}\right\}$ generated by their method R-linearly converges to $x^{*} \in \Omega^{*}$, where $\Omega^{*}$ is the solution set of (1.1). Other gradient-based projection methods for large-scale nonlinear monotone equations can be found in $[11-15,18,23,26,33,35]$.

Motivated by the works of $[19,30]$, we propose a descent Perry-type derivative-free projection method for solving large-scale nonlinear monotone equations. This method is presented in the next section. In Section 3, we prove the global convergence of the proposed method followed by the convergence rate in Section 4 . Numerical results follow in Section 5. Finally, conclusion is presented in Section 6.

\section{Motivation And the Algorithm}

In this section, we describe the details of the proposed method. But first, we briefly review the work of Livieris and Pintelas [19] and that of Yao and Ning [30] which motivated this work.

Livieris and Pintelas, in [19], proposed a modified Perry's conjugate gradient method for the unconstrained optimization problem

$$
\min \left\{f(x) \mid x \in \mathbb{R}^{n}\right\},
$$

with $f: \mathbb{R}^{n} \rightarrow \mathbb{R}$ being a continuously differentiable function bounded from below. The method generates iterations $x_{k+1}=x_{k}+\alpha_{k} d_{k}$ using the direction

$$
d_{k}= \begin{cases}-g_{k}, & \text { if } k=0 \\ -\left(1+\beta_{k}^{M P} \frac{g_{k}^{T} d_{k-1}}{\left\|g_{k}\right\|^{2}}\right) g_{k}+\beta_{k}^{M P} d_{k-1}, & \text { if } k \geq 1\end{cases}
$$

where $g_{k}=\nabla f\left(x_{k}\right)$ is the gradient of $f$ at $x_{k}$, and

$$
\beta_{k}^{M P}=\frac{g_{k}^{T}\left(w_{k-1}-s_{k-1}\right)}{w_{k-1}^{T} d_{k-1}}
$$

with

$$
w_{k-1}=y_{k-1}+h_{k}\left\|g_{k-1}\right\|^{r} s_{k-1} .
$$


The authors suggested $h_{k}$ be given as

$$
h_{k}=t+\max \left\{-\frac{s_{k-1}^{T} y_{k-1}}{\left\|s_{k-1}\right\|^{2}}, 0\right\}\left\|g_{k-1}\right\|^{-r},
$$

where $t$ and $r$ are positive constants, and $y_{k-1}=g_{k}-g_{k-1}$ and $s_{k-1}=x_{k}-x_{k-1}$. The parameter $\beta_{k}^{\mathrm{MP}}$ was shown to satisfy the sufficient descent property

$$
d_{k}^{T} g_{k} \leq-c\left\|g_{k}\right\|^{2}, \quad \forall k \geq 0,
$$

where $c>0$ is a positive constant and that the method is globally convergent.

Yao and Ning [30], using a modified symmetric Perry matrix

$$
Q_{k}=I-t_{k} \frac{s_{k-1} y_{k-1}^{T}+y_{k-1} s_{k-1}^{T}}{s_{k-1}^{T} y_{k-1}}+\frac{s_{k-1} s_{k-1}^{T}}{s_{k-1}^{T} y_{k-1}},
$$

where $t_{k}$ is a positive parameter to be determined, defined their search direction as

$$
d_{k}=-Q_{k} g_{k}, \forall k \geq 1 .
$$

By minimizing the distance, in the Frobenius norm, between the above Perry matrix and the self-scaling memoryless BFGS matrix [24]

$$
H=\xi_{k} I-\xi_{k} \frac{s_{k-1} y_{k-1}^{T}+y_{k-1} s_{k-1}^{T}}{s_{k-1}^{T} y_{k-1}}+\left(1+\xi_{k} \frac{\left\|y_{k-1}\right\|^{2}}{s_{k-1}^{T} y_{k-1}}\right) \frac{s_{k-1} s_{k-1}^{T}}{s_{k-1}^{T} y_{k-1}},
$$

they determined an optimal parameter $t_{k}^{*}$ as

$$
t_{k}^{*}=\frac{1}{1+a_{k}}, \quad a_{k}=\frac{\left\|s_{k-1}\right\|^{2}\left\|y_{k-1}\right\|^{2}}{\left(s_{k-1}^{T} y_{k-1}\right)^{2}} .
$$

Hence, using this optimal parameter $t_{k}^{*}$, the authors suggested an adaptive three term Perry's conjugate gradient method

$$
d_{k}=-g_{k}+\beta_{k} d_{k-1}+\delta_{k} y_{k-1}
$$

where

$$
\beta_{k}=\frac{g_{k}^{T}\left(t_{k} y_{k-1}-s_{k-1}\right)}{d_{k-1}^{T} y_{k-1}} \text { and } \delta_{k}=\frac{t_{k} g_{k}^{T} s_{k-1}}{s_{k-1}^{T} y_{k-1}}
$$

with

$$
t_{k}=\min \left\{\frac{1}{1+a_{k}}, \frac{s_{k-1}^{T} y_{k-1}}{\left\|y_{k-1}\right\|^{2}}\right\} .
$$

The method was shown to be effective numerically.

Now, motivated by the above discussed modified Perry's conjugate gradient methods of $[19,30]$, we propose a modified Perry-type derivative-free projection method for solving (1.1). By taking a careful look at the search direction presented in [19] and the $\beta_{k}$ parameter in [30], we propose our search direction as

$$
d_{k}= \begin{cases}-F_{k}, & \text { if } k=0, \\ -\left(\lambda_{k}+\beta_{k}^{M} \frac{F_{k}^{T} d_{k-1}}{\left\|F_{k}\right\|^{2}}\right) F_{k}+\beta_{k}^{M} d_{k-1}, & \text { if } k \geq 1,\end{cases}
$$


where

$$
\beta_{k}^{M}=\frac{F_{k}^{T}\left(\lambda_{k} w_{k-1}-s_{k-1}\right)}{w_{k-1}^{T} d_{k-1}} \quad \text { and } \quad \lambda_{k}= \begin{cases}\lambda^{*}, & \text { if } \lambda^{*} \in[\kappa, 1] \\ 1, & \text { otherwise }\end{cases}
$$

with $\lambda^{*}=\frac{\left\|s_{k-1}\right\|^{2}}{s_{k-1}^{T} u_{k-1}}, s_{k-1}=z_{k-1}-x_{k-1}, u_{k-1}=y_{k-1}+\phi s_{k-1}, y_{k-1}=F\left(z_{k-1}\right)-F_{k-1}, w_{k-1}=u_{k-1}+$ $\left\|F_{k-1}\right\| s_{k-1}$, and $\phi$ is a positive constant and $\kappa \in(0,1]$. With $d_{k}$ given in (1.3) above, we determine the next iterate $x_{k+1}$ using (1.2), where the step length $\alpha_{k}=\max \left\{\rho^{i}: i=0,1,2, \ldots\right\}, \rho \in(0,1]$, is such that it satisfies

$$
-F\left(x_{k}+\alpha_{k} d_{k}\right)^{T} d_{k} \geq \sigma \alpha_{k}\left\|F\left(x_{k}+\alpha_{k} d_{k}\right)\right\|\left\|d_{k}\right\|^{2}, \quad \sigma>0 .
$$

We present our proposed method below.

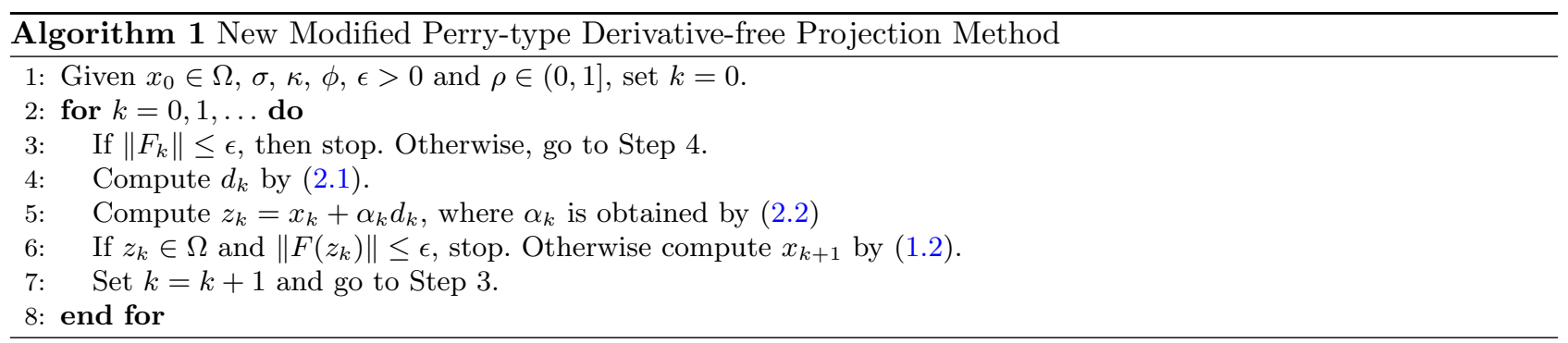

\section{Global Convergence}

We now establish the global convergence of the presented Algorithm 1. Throughout the paper, we assume that $F_{k} \neq 0$ for all $k$, otherwise a stationary point has been found. We also assume that the following assumption holds.

\section{Assumption 3.1.}

(i) The function $F(\cdot)$ is monotone on $\mathbb{R}^{n}$.

(ii) The solution set $\Omega^{*}$ is nonempty.

(iii) The function $F(\cdot)$ is Lipschitz continuous on $\mathbb{R}^{n}$, i.e. there exists a positive constant $L$ such that

$$
\|F(x)-F(y)\| \leq L\|x-y\|, \quad \forall x, y \in \mathbb{R}^{n} .
$$

Note that from the monotonicity of $F$, we have that

$$
s_{k-1}^{T} u_{k-1}=\left(F\left(z_{k-1}\right)-F_{k-1}\right)^{T}\left(z_{k-1}-x_{k-1}\right)+\phi\left\|s_{k-1}\right\|^{2} \geq \phi\left\|s_{k-1}\right\|^{2}>0 .
$$

This indicates that $\lambda^{*}$ is positive whenever $s_{k-1}$ is not zero and hence $\lambda_{k}$ in (2.1) is well-defined.

Lemma 3.2. The search direction $d_{k}$ generated by Algorithm 1 satisfies the descent condition

$$
F_{k}^{T} d_{k} \leq-\kappa\left\|F_{k}\right\|, \quad \forall k \geq 0 \text { and } \kappa>0 .
$$

Proof. Since $d_{0}=-F_{0}$, we have $F_{0}^{T} d_{0}=-\left\|F_{0}\right\|^{2}$, which satisfies (3.1). Now, for $k \geq 1$, we obtain from (2.1) and the relation $\lambda_{k} \geq \kappa$ that

$$
\begin{aligned}
F_{k}^{T} d_{k} & =-\left(\lambda_{k}+\beta_{k}^{M} \frac{F_{k}^{T} d_{k-1}}{\left\|F_{k}\right\|^{2}}\right)\left\|F_{k}\right\|^{2}+\beta_{k}^{M} F_{k}^{T} d_{k-1} \\
& =-\lambda_{k}\left\|F_{k}\right\|^{2} \\
& \leq-\kappa\left\|F_{k}\right\|^{2}
\end{aligned}
$$


Lemma 3.3. Suppose that Assumption 3.1 holds, and let the sequences $\left\{x_{k}\right\}$ and $\left\{z_{k}\right\}$ be generated by Algorithm 1. Then, for any $x^{*} \in \Omega$, it holds that

$$
\left\|x_{k+1}-x^{*}\right\|^{2} \leq\left\|x_{k}-x^{*}\right\|^{2}-\sigma^{2}\left\|x_{k}-z_{k}\right\|^{4} .
$$

Moreover, the sequences $\left\{x_{k}\right\}$ and $\left\{z_{k}\right\}$ are bounded, and

$$
\sum_{k=0}^{\infty}\left\|x_{k}-z_{k}\right\|^{4}<\infty
$$

Furthermore, it holds that

$$
\lim _{k \rightarrow \infty} \alpha_{k}\left\|d_{k}\right\|=0
$$

Proof. From (2.2), we have

$$
F\left(z_{k}\right)^{T}\left(x_{k}-z_{k}\right) \geq \sigma\left\|F\left(z_{k}\right)\right\|\left\|x_{k}-z_{k}\right\|^{2}>0 .
$$

For $x^{*} \in \Omega$, we obtain from (1.2) that

$$
\begin{aligned}
\left\|x_{k+1}-x^{*}\right\|^{2} & =\left\|P_{\Omega}\left(x_{k}-\theta_{k} F\left(z_{k}\right)\right)-x^{*}\right\|^{2} \\
& \leq\left\|x_{k}-\theta_{k} F\left(z_{k}\right)-x^{*}\right\|^{2} \\
& =\left\|x_{k}-x^{*}\right\|^{2}-2 \theta_{k} F\left(z_{k}\right)^{T}\left(x_{k}-x^{*}\right)+\theta_{k}^{2}\left\|F\left(z_{k}\right)\right\|^{2},
\end{aligned}
$$

where $\theta_{k}=\frac{F\left(z_{k}\right)^{T}\left(x_{k}-z_{k}\right)}{\left\|F\left(z_{k}\right)\right\|^{2}}$. By monotonicity of $F$, we obtain

$$
\begin{aligned}
F\left(z_{k}\right)^{T}\left(x_{k}-x^{*}\right) & =F\left(z_{k}\right)^{T}\left(x_{k}-z_{k}\right)+F\left(z_{k}\right)^{T}\left(z_{k}-x^{*}\right) \\
& \geq F\left(z_{k}\right)^{T}\left(x_{k}-z_{k}\right)+F\left(x^{*}\right)^{T}\left(z_{k}-x^{*}\right) \\
& =F\left(z_{k}\right)^{T}\left(x_{k}-z_{k}\right) .
\end{aligned}
$$

From (3.4)-(3.6), we get that

$$
\begin{aligned}
\left\|x_{k+1}-x^{*}\right\|^{2} & \leq\left\|x_{k}-x^{*}\right\|^{2}-2 \theta_{k} F\left(z_{k}\right)^{T}\left(x_{k}-z_{k}\right)+\theta_{k}^{2}\left\|F\left(z_{k}\right)\right\|^{2} \\
& =\left\|x_{k}-x^{*}\right\|^{2}-\frac{\left(F\left(z_{k}\right)^{T}\left(x_{k}-z_{k}\right)\right)^{2}}{\left\|F\left(z_{k}\right)\right\|^{2}} \\
& \leq\left\|x_{k}-x^{*}\right\|^{2}-\sigma^{2}\left\|x_{k}-z_{k}\right\|^{4} .
\end{aligned}
$$

Thus, the sequence $\left\{\left\|x_{k}-x^{*}\right\|\right\}$ is decreasing and convergent, and hence $\left\{x_{k}\right\}$ is bounded. From (3.4), it follows that

$$
\begin{aligned}
\sigma\left\|F\left(z_{k}\right)\right\|\left\|x_{k}-z_{k}\right\|^{2} & \leq F\left(z_{k}\right)^{T}\left(x_{k}-z_{k}\right) \\
& \leq\left\|F\left(z_{k}\right)\right\|\left\|x_{k}-z_{k}\right\|,
\end{aligned}
$$

giving

$$
\sigma\left\|x_{k}-z_{k}\right\| \leq 1
$$

which shows that $\left\{z_{k}\right\}$ is also bounded. 
Furthermore, it follows from (3.7) that

$$
\sigma^{2} \sum_{k=0}^{\infty}\left\|x_{k}-z_{k}\right\|^{4} \leq \sum_{k=0}^{\infty}\left(\left\|x_{k}-x^{*}\right\|^{2}-\left\|x_{k+1}-x^{*}\right\|^{2}\right)<\infty,
$$

which implies

$$
\lim _{k \rightarrow \infty}\left\|x_{k}-z_{k}\right\|=\lim _{k \rightarrow \infty} \alpha_{k}\left\|d_{k}\right\|=0
$$

Observe from (3.7) that

$$
\left\|x_{k+1}-x^{*}\right\|^{2} \leq\left\|x_{k}-x^{*}\right\|^{2}
$$

implies

$$
\left\|x_{k}-x^{*}\right\|^{2} \leq\left\|x_{0}-x^{*}\right\|^{2}, \quad \forall k \geq 0 .
$$

Therefore, since $F$ is continuous, by the Lipschitz condition we get that

$$
\begin{aligned}
\left\|F\left(x_{k}\right)\right\| & =\left\|F\left(x_{k}\right)-F\left(x^{*}\right)\right\| \\
& \leq L\left\|x_{k}-x^{*}\right\| \\
& \leq L\left\|x_{0}-x^{*}\right\| .
\end{aligned}
$$

Taking $\gamma=L\left\|x_{0}-x^{*}\right\|$ gives that $\left\|F\left(x_{k}\right)\right\| \leq \gamma$. Also, (3.8) implies that there is a positive constant $\mu$ such that $\left\|s_{k}\right\| \leq \mu, k \geq 0$.

Lemma 3.4. For all $k \geq 0$, we have

$$
\kappa\left\|F_{k}\right\| \leq\left\|d_{k}\right\| \leq \varphi\left\|F_{k}\right\| .
$$

where $\varphi$ is a positive constant.

Proof. From (3.1) and Cauchy-Schwarz inequality, we obtain

$$
\left\|d_{k}\right\| \geq \kappa\left\|F_{k}\right\|
$$

And from (2.1), we have

$$
\left\|d_{k}\right\| \leq\left\|F_{k}\right\|+2\left|\beta_{k}^{M}\right| \mid d_{k-1} \| .
$$

From the definitions of $u_{k-1}, w_{k-1}$ and $s_{k-1}$ in (2.1), we get that there exist positive constants $\varpi$ and $\varrho$ such that

$$
\left\|u_{k-1}\right\| \leq\left\|F\left(z_{k}\right)\right\|+\left\|F_{k-1}\right\|+\phi\left\|s_{k-1}\right\| \leq 2 \gamma+\phi \mu=\varpi
$$

and

$$
\left\|w_{k-1}\right\| \leq\left\|u_{k-1}\right\|+\left\|F_{k-1}\right\|\left\|s_{k-1}\right\| \leq \varpi+\gamma \mu=\varrho .
$$

Notice that for $F_{k-1} \neq 0$ and $s_{k-1} \neq 0$, we get

$$
s_{k-1}^{T} w_{k-1}=s_{k-1}^{T} u_{k-1}+\left\|F_{k-1}\right\|\left\|s_{k-1}\right\|^{2}>\left\|F_{k-1}\right\|\left\|s_{k-1}\right\|^{2}>0 .
$$


Hence, there is a constant $\omega>0$ such that

$$
d_{k-1}^{T} w_{k-1}=\alpha_{k-1}^{-1} s_{k-1}^{T} w_{k-1}>\alpha_{k-1}^{-1}\left\|F_{k-1}\right\|\left\|s_{k-1}\right\|^{2} \geq \omega\left\|d_{k-1}\right\| .
$$

It then follows that

$$
\left|\beta_{k}^{M}\right| \leq \frac{\left\|F_{k}\right\|\left(\left\|w_{k-1}\right\|+\left\|s_{k-1}\right\|\right)}{w_{k-1}^{T} d_{k-1}} \leq \frac{\left\|F_{k}\right\|(\varrho+\mu)}{\omega\left\|d_{k-1}\right\|} .
$$

From (3.10) we get

$$
\left\|d_{k}\right\| \leq\left\|F_{k}\right\|+2 \frac{\left\|F_{k}\right\|(\varrho+\mu)}{\omega\left\|d_{k-1}\right\|}\left\|d_{k-1}\right\|=\left\|F_{k}\right\|\left(1+\frac{2(\varrho+\mu)}{\omega}\right)=\varphi\left\|F_{k}\right\|,
$$

where $\varphi=1+\frac{2(\varrho+\mu)}{\omega}$.

Lemma 3.5. Let $\left\{x_{k}\right\}$ and $\left\{z_{k}\right\}$ be generated by Algorithm 1. Then

$$
\alpha_{k} \geq \min \left\{1, \frac{\kappa \rho}{(L+\sigma \gamma) \varphi^{2}}\right\}>0 .
$$

Proof. From the line search procedure (2.2), if $\alpha_{k} \neq 1$, then $\alpha_{k}^{\prime}=\rho^{-1} \alpha_{k}$ does not satisfy (2.2). This means that

$$
-F\left(z_{k}^{\prime}\right)^{T} d_{k}<\sigma \alpha_{k}^{\prime}\left\|F\left(z_{k}^{\prime}\right)\right\|\left\|d_{k}\right\|^{2}
$$

where $z_{k}^{\prime}=x_{k}+\alpha_{k}^{\prime} d_{k}$. This together with (2.2), (3.1) and (3.9) imply that

$$
\begin{aligned}
\kappa\left\|F_{k}\right\|^{2} & \leq-F_{k}^{T} d_{k} \\
& =\left(F\left(z_{k}^{\prime}\right)-F_{k}\right)^{T} d_{k}-F\left(z_{k}^{\prime}\right)^{T} d_{k} \\
& \leq L \alpha_{k}^{\prime}\left\|d_{k}\right\|^{2}+\sigma \alpha_{k}^{\prime}\left\|F\left(z_{k}^{\prime}\right)\right\|\left\|d_{k}\right\|^{2} \\
& =\left(L+\sigma\left\|F\left(z_{k}^{\prime}\right)\right\|\right) \alpha_{k} \rho^{-1}\left\|d_{k}\right\|^{2} \\
& \leq(L+\sigma \gamma) \alpha_{k} \rho^{-1} \varphi^{2}\left\|F_{k}\right\|^{2} .
\end{aligned}
$$

Thus

$$
\alpha_{k} \geq \frac{\kappa \rho}{(L+\sigma \gamma) \varphi^{2}}>0
$$

which gives the desired result.

Theorem 3.6. Suppose that Assumption 3.1 holds, and let the sequence $\left\{x_{k}\right\}$ be generated by Algorithm 1 . Then

$$
\liminf _{k \rightarrow \infty}\left\|F_{k}\right\|=0 .
$$

Proof. We assume that (3.12) does not hold, that is, $\exists \eta>0$ such that $\left\|F_{k}\right\| \geq \eta, \forall k \geq 0$. It then follows from (3.9) that

$$
\left\|d_{k}\right\| \geq \kappa\left\|F_{k}\right\| \geq \kappa \eta>0, \quad \forall k \geq 0,
$$

and (3.3) implies that

$$
\lim _{k \rightarrow \infty} \alpha_{k}=0
$$

On the other hand, (3.11) implies that $\alpha_{k}>0, \forall k \geq 0$, which contradicts (3.13). Thus, (3.12) holds. 


\section{Convergence Rate}

From the above discussions, it is evident that the sequence $\left\{x_{k}\right\}$ converges to a solution of problem (1.1). Therefore, we always assume that $x_{k}$ converges to $x^{*}$ as $k \rightarrow \infty$, where $x^{*}$ belongs to the solution set $\Omega^{*}$ of problem (1.1). To determine the rate of convergence for the proposed algorithm, we also assume the following assumption holds.

Assumption 4.1. For any $x^{*} \in \Omega^{*}$, there exist two positive constants $\psi$ and $\delta$ satisfying

$$
\psi \operatorname{dist}\left(x, \Omega^{*}\right) \leq\|F(x)\|^{2}, \quad \forall x \in \mathcal{N}_{\delta}\left(x^{*}\right),
$$

where $\mathcal{N}_{\delta}\left(x^{*}\right)=\left\{x \in \mathbb{R}^{n}:\left\|x-x^{*}\right\| \leq \delta\right\}$ and $\operatorname{dist}\left(x, \Omega^{*}\right)$ is the distance from $x$ to $\Omega^{*}$.

Theorem 4.2. Let Assumptions 3.1 and 4.1 hold, and the sequence $\left\{x_{k}\right\}$ be generated by Algorithm 1. Then the sequence $\left\{\operatorname{dist}\left(x_{k}, \Omega^{*}\right)\right\}$ is $Q$-linearly convergent to 0 , and hence the sequence $\left\{x_{k}\right\}$ R-linearly converges to $x^{*}$.

Proof. Let $\bar{x}_{k}:=\arg \min \left\{\left\|x_{k}-\bar{x}\right\|: \bar{x} \in \Omega^{*}\right\}$, which implies that $\bar{x}_{k}$ is the closest solution to $x_{k}$, namely,

$$
\left\|x_{k}-\bar{x}_{k}\right\|=\operatorname{dist}\left(x_{k}, \Omega^{*}\right) .
$$

Denoting $x^{*}$ by $\bar{x}_{k}$, it follows from (3.2) that

$$
\left\|x_{k+1}-\bar{x}_{k}\right\|^{2} \leq\left\|x_{k}-\bar{x}_{k}\right\|^{2}-\sigma^{2}\left\|x_{k}-z_{k}\right\|^{4} .
$$

This, together with (3.9) and (4.1), give that for $\bar{x}_{k} \in \Omega^{*}$,

$$
\begin{aligned}
\operatorname{dist}\left(x_{k+1}, \Omega^{*}\right)^{2} & =\left\|x_{k+1}-\bar{x}_{k}\right\|^{2} \\
& \leq\left\|x_{k}-\bar{x}_{k}\right\|^{2}-\sigma^{2}\left\|x_{k}-z_{k}\right\|^{4} \\
& \leq \operatorname{dist}\left(x_{k}, \Omega^{*}\right)^{2}-\sigma^{2}\left\|\alpha_{k} d_{k}\right\|^{4} \\
& \leq \operatorname{dist}\left(x_{k}, \Omega^{*}\right)^{2}-\sigma^{2} \kappa^{4} \alpha_{k}^{4}\left\|F_{k}\right\|^{4} \\
& \leq \operatorname{dist}\left(x_{k}, \Omega^{*}\right)^{2}-\sigma^{2} \psi^{2} \kappa^{4} \alpha_{k}^{4} \operatorname{dist}\left(x_{k}, \Omega^{*}\right)^{2} \\
& =\left(1-\sigma^{2} \psi^{2} \kappa^{4} \alpha_{k}^{4}\right) \operatorname{dist}\left(x_{k}, \Omega^{*}\right)^{2} .
\end{aligned}
$$

Taking $\kappa^{2} \leq \frac{1}{\sigma \psi}$, we get that $1-\sigma^{2} \psi^{2} \kappa^{4} \alpha_{k}^{4} \in(0,1)$ holds. This implies that the sequence $\left\{\operatorname{dist}\left(x_{k}, \Omega^{*}\right)\right\}$ converges to 0 Q-linearly. Therefore, the whole sequence $\left\{x_{k}\right\}$ converges to $x^{*} \mathrm{R}$-linearly.

\section{NumericAl EXPERIMENTS}

In this section, we report some numerical results to test the efficacy of our proposed Algorithm 1, herein denoted as NMPCG. We compare it with other three term derivative-free projection methods that have recently been proposed in the literature. These are the three-term conjugate descent projection method of Abubakar et al. [2], denoted M3TCD1, the derivative-free iterative method of Liu and Feng [16], denoted $P D Y$, and the partially symmetrical derivative-free Liu-Storey projection method of Liu et al. [18], denoted $s L S$. All algorithms are coded in MATLAB R2019b and the methods are compared using number of iterations, number of function evaluations and CPU time taken for each method to reach the optimal value or termination. We test the algorithms on eight test problems, with various dimensions, using four different starting points $x_{0}^{1}=(-0.1,-0.1, \ldots,-0.1)^{T}, x_{0}^{2}=(0.1,0.1, \ldots, 0.1)^{T}, x_{0}^{3}=(0.5,0.5, \ldots, 0.5)^{T}$ and $x_{0}^{4}=(2,2, \ldots, 2)^{T}$. The eight test problems, where the mapping $F(\cdot)$ is taken as $F(x)=\left(F_{1}(x), F_{2}(x), F_{3}(x), \ldots, F_{n}(x)\right)^{T}$, are as follows.

Problem 1 [2].

$$
F_{i}(x)=e^{x_{i}}-1, \quad \text { for } \quad i=1,2,3, \ldots, n \text { and } \Omega=\mathbb{R}_{+}^{n} .
$$


Problem 2 [2].

$$
\begin{aligned}
& F_{1}(x)=x_{1}-e^{\cos \left(\frac{x_{1}+x_{2}}{n+1}\right)}, \\
& F_{i}(x)=x_{i}-e^{\cos \left(\frac{x_{i-1}+x_{i}+x_{i+1}}{n+1}\right)}, \quad \text { for } \quad i=2,3, \ldots, n-1 \quad \text { and } \quad \Omega=\mathbb{R}_{+}^{n}, \\
& F_{n}(x)=x_{n}-e^{\cos \left(\frac{x_{n-1}+x_{n}}{n+1}\right)} .
\end{aligned}
$$

Problem 3 [2].

$$
F_{i}(x)=2 x_{i}-\sin \left(\left|x_{i}\right|\right), \quad \text { for } \quad i=1,2,3, \ldots, n \quad \text { and } \quad \Omega=\mathbb{R}_{+}^{n}
$$

Problem 4 [13].

$$
F_{i}(x)=\ln \left(\left|x_{i}\right|+1\right)-\frac{x_{i}}{n}, \quad \text { for } \quad i=1,2,3, \ldots, n \quad \text { and } \quad \Omega=\mathbb{R}_{+}^{n} .
$$

Problem 5 [2].

$$
F_{i}(x)=x_{i}-\sin \left(\left|x_{i}-1\right|\right), \quad \text { for } \quad i=1,2, \ldots, n \quad \text { and } \quad \Omega=\left\{x \in \mathbb{R}^{n}: \sum_{i=1}^{n} x_{i} \leq n, x_{i} \geq 0\right\} .
$$

Problem 6. [2].

$$
F_{i}(x)=\ln \left(x_{i}+1\right)-\frac{x_{i}}{n}, \quad \text { for } \quad i=1,2,3, \ldots, n \quad \text { and } \quad \Omega=\left\{x \in \mathbb{R}^{n}: \sum_{i=1}^{n} x_{i} \leq n, x_{i}>-1\right\} .
$$

Problem 7 [11].

$$
\begin{aligned}
F_{1}(x) & =x_{1}\left(2 x_{1}^{2}+2 x_{2}^{2}\right)-1 \\
F_{i}(x) & =x_{i}\left(x_{i-1}^{2}+2 x_{i}^{2}+x_{i+1}^{2}\right)-1, \quad \text { for } \quad i=2,3, \ldots, n-1 \quad \text { and } \quad \Omega=\mathbb{R}_{+}^{n}, \\
F_{n}(x) & =x_{n}\left(2 x_{n-1}^{2}+2 x_{n}^{2}\right)-1 .
\end{aligned}
$$

Problem 8 [17].

$$
\begin{aligned}
& F_{1}(x)=x_{1}-e^{\cos \left(\frac{x_{1}+x_{2}}{2}\right)}, \\
& F_{i}(x)=x_{i}-e^{\cos \left(\frac{x_{i-1}+x_{i}+x_{i+1}}{i}\right)}, \quad \text { for } \quad i=2,3, \ldots, n-1 \quad \text { and } \quad \Omega=\mathbb{R}_{+}^{n}, \\
& F_{n}(x)=x_{n}-e^{\cos \left(\frac{x_{n-1}+x_{n}}{n}\right)} .
\end{aligned}
$$

In our experiments, the algorithms are stopped whenever the inequality $\left\|F_{k}\right\| \leq \epsilon=10^{-6}$ is satisfied, or the total number of iterations exceeds 1000. The NMPCG method is implemented with the parameters $\sigma=10^{-4}$, $\rho=0.5, \phi=10^{-5}$ and $\kappa=10^{-5}$, while parameters for the algorithms $s L S, P D Y$ and $M 3 T C D 1$ are set as in respective papers.

The numerical results are reported in Tables 1 and 2, where ITER refers to the number of iterations, FE stands for the number of function evaluations and $C P U$ is the CPU time in seconds. We note here that all algorithms managed to solve all the eight test problems successfully. From Tables 1 and 2, we see that in terms of number of iterations, the $N M P C G$ and $M 3 T C D 1$ methods are very competitive for most problems, and perform much better than the other methods. However, in terms of function evaluations, we see that the proposed $N M P C G$ method is the best. Overall, the results indicate that the proposed NMPCG method performs better than the other competing methods, with the $s L S$ method the weaker one among the four competing methods. 
TABLE 1. Numerical results for problem 1-4.

\begin{tabular}{|c|c|c|c|c|c|c|c|c|c|c|c|c|c|c|}
\hline & & & & ITER & & & & $\mathrm{FE}$ & & & & $\mathrm{CPU}$ & & \\
\hline PROB & $x_{0}$ & DIM & NMPCG & PDY & M3TCD1 & sLS & NMPCG & PDY & M3TCD1 & sLS & NMPCG & PDY & M3TCD1 & sLS \\
\hline \multirow[t]{16}{*}{1} & \multirow[t]{4}{*}{$x_{0}^{1}$} & 5000 & 1 & 1 & 1 & 1 & 3 & 3 & 3 & 3 & 0.0015 & 0.0011 & 0.0020 & 0.0016 \\
\hline & & 10000 & 1 & 1 & 1 & 1 & 3 & 3 & 3 & 3 & 0.0045 & 0.0010 & 0.0021 & 0.0007 \\
\hline & & 20000 & 1 & 1 & 1 & 1 & 3 & 3 & 3 & 3 & 0.0009 & 0.0009 & 0.0009 & 0.0015 \\
\hline & & 50000 & 1 & 1 & 1 & 1 & 3 & 3 & 3 & 3 & 0.0026 & 0.0033 & 0.0034 & 0.0031 \\
\hline & \multirow[t]{4}{*}{$x_{0}^{2}$} & 5000 & 6 & 16 & 8 & 22 & 11 & 45 & 21 & 58 & 0.0039 & 0.0114 & 0.0063 & 0.0125 \\
\hline & & 10000 & 6 & 16 & 8 & 22 & 11 & 45 & 21 & 58 & 0.0031 & 0.0105 & 0.0051 & 0.0177 \\
\hline & & 20000 & 6 & 16 & 8 & 23 & 11 & 45 & 21 & 60 & 0.0069 & 0.0194 & 0.0099 & 0.0301 \\
\hline & & 50000 & 6 & 17 & 9 & 23 & 11 & 48 & 24 & 60 & 0.0106 & 0.0351 & 0.0170 & 0.0573 \\
\hline & \multirow[t]{4}{*}{$x_{0}^{3}$} & 5000 & 7 & 17 & 9 & 23 & 13 & 48 & 26 & 61 & 0.0022 & 0.0071 & 0.0035 & 0.0100 \\
\hline & & 10000 & 7 & 17 & 9 & 23 & 13 & 48 & 26 & 61 & 0.0035 & 0.0103 & 0.0054 & 0.0157 \\
\hline & & 20000 & 7 & 18 & 9 & 24 & 13 & 51 & 26 & 63 & 0.0057 & 0.0233 & 0.0086 & 0.0276 \\
\hline & & 50000 & 7 & 18 & 9 & 24 & 13 & 51 & 26 & 62 & 0.0169 & 0.0546 & 0.0210 & 0.0559 \\
\hline & \multirow{4}{*}{$x_{0}^{4}$} & 5000 & 8 & 19 & 8 & 23 & 16 & 57 & 32 & 63 & 0.0025 & 0.0078 & 0.0035 & 0.0105 \\
\hline & & 10000 & 8 & 19 & 9 & 24 & 16 & 57 & 35 & 65 & 0.0042 & 0.0117 & 0.0067 & 0.0147 \\
\hline & & 20000 & 8 & 21 & 9 & 24 & 16 & 69 & 35 & 64 & 0.0061 & 0.0221 & 0.0100 & 0.0239 \\
\hline & & 50000 & 8 & 22 & 9 & 25 & 16 & 76 & 35 & 67 & 0.0139 & 0.0506 & 0.0214 & 0.0569 \\
\hline \multirow[t]{16}{*}{2} & \multirow[t]{4}{*}{$x_{0}^{1}$} & 5000 & 7 & 19 & 10 & 27 & 15 & 54 & 27 & 69 & 0.0085 & 0.0280 & 0.0147 & 0.0304 \\
\hline & & 10000 & 5 & 21 & 10 & 27 & 10 & 64 & 27 & 69 & 0.0084 & 0.0628 & 0.0260 & 0.0693 \\
\hline & & 20000 & 7 & 22 & 10 & 28 & 14 & 68 & 27 & 71 & 0.0239 & 0.1092 & 0.0489 & 0.1142 \\
\hline & & 50000 & 6 & 25 & 10 & 28 & 12 & 86 & 27 & 69 & 0.0452 & 0.3695 & 0.1014 & 0.2640 \\
\hline & \multirow[t]{4}{*}{$x_{0}^{2}$} & 5000 & 7 & 19 & 10 & 27 & 15 & 54 & 27 & 69 & 0.0066 & 0.0291 & 0.0114 & 0.0378 \\
\hline & & 10000 & 5 & 21 & 10 & 27 & 10 & 64 & 27 & 69 & 0.0087 & 0.0648 & 0.0217 & 0.0619 \\
\hline & & 20000 & 5 & 21 & 10 & 27 & 10 & 64 & 27 & 68 & 0.0160 & 0.1236 & 0.0452 & 0.1105 \\
\hline & & 50000 & 6 & 24 & 10 & 28 & 12 & 81 & 27 & 69 & 0.0453 & 0.2935 & 0.1106 & 0.3002 \\
\hline & \multirow[t]{4}{*}{$x_{0}^{3}$} & 5000 & 7 & 18 & 10 & 26 & 15 & 51 & 27 & 66 & 0.0065 & 0.0253 & 0.0120 & 0.0372 \\
\hline & & 10000 & 5 & 20 & 10 & 27 & 10 & 59 & 27 & 69 & 0.0105 & 0.0475 & 0.0248 & 0.0583 \\
\hline & & 20000 & 5 & 21 & 10 & 27 & 10 & 64 & 27 & 69 & 0.0192 & 0.1125 & 0.0524 & 0.1204 \\
\hline & & 50000 & 6 & 23 & 10 & 28 & 12 & 76 & 27 & 69 & 0.0465 & 0.2943 & 0.1008 & 0.2811 \\
\hline & $x_{0}^{4}$ & 5000 & 5 & 17 & 9 & 25 & 10 & 48 & 24 & 65 & 0.0056 & 0.0198 & 0.0116 & 0.0320 \\
\hline & & 10000 & 5 & 18 & 9 & 25 & 10 & 51 & 24 & 65 & 0.0089 & 0.0413 & 0.0196 & 0.0554 \\
\hline & & 20000 & 5 & 18 & 10 & 26 & 10 & 51 & 27 & 67 & 0.0158 & 0.0916 & 0.0417 & 0.1228 \\
\hline & & 50000 & 5 & 18 & 10 & 26 & 10 & 51 & 27 & 65 & 0.0374 & 0.2202 & 0.0981 & 0.2540 \\
\hline 3 & $x_{0}^{1}$ & 5000 & 1 & 1 & 1 & 1 & 5 & 5 & 14 & 5 & 0.0005 & 0.0004 & 0.0011 & 0.0005 \\
\hline & & 10000 & 1 & 1 & 1 & 1 & 5 & 5 & 14 & 5 & 0.0007 & 0.0006 & 0.0016 & 0.0006 \\
\hline & & 20000 & 1 & 1 & 1 & 1 & 5 & 5 & 14 & 5 & 0.0011 & 0.0011 & 0.0030 & 0.0011 \\
\hline & & 50000 & 1 & 1 & 1 & 1 & 5 & 5 & 14 & 5 & 0.0023 & 0.0024 & 0.0061 & 0.0023 \\
\hline & $x_{0}^{2}$ & 5000 & 5 & 16 & 8 & 22 & 9 & 45 & 21 & 58 & 0.0013 & 0.0051 & 0.0023 & 0.0074 \\
\hline & & 10000 & 5 & 16 & 8 & 22 & 9 & 45 & 21 & 57 & 0.0018 & 0.0076 & 0.0036 & 0.0122 \\
\hline & & 20000 & 5 & 16 & 9 & 22 & 9 & 45 & 24 & 57 & 0.0032 & 0.0136 & 0.0069 & 0.0207 \\
\hline & & 50000 & 5 & 17 & 9 & 22 & 9 & 48 & 24 & 56 & 0.0071 & 0.0292 & 0.0144 & 0.0437 \\
\hline & $x_{0}^{3}$ & 5000 & 5 & 17 & 9 & 24 & 9 & 48 & 24 & 62 & 0.0013 & 0.0059 & 0.0029 & 0.0088 \\
\hline & & 10000 & 5 & 17 & 9 & 25 & 9 & 48 & 24 & 64 & 0.0020 & 0.0087 & 0.0044 & 0.0136 \\
\hline & & 20000 & 5 & 18 & 9 & 25 & 9 & 51 & 23 & 64 & 0.0032 & 0.0152 & 0.0065 & 0.0230 \\
\hline & & 50000 & 6 & 18 & 9 & 25 & 11 & 51 & 24 & 63 & 0.0086 & 0.0309 & 0.0145 & 0.0483 \\
\hline & $x_{0}^{4}$ & 5000 & 6 & 19 & 10 & 22 & 11 & 56 & 31 & 58 & 0.0016 & 0.0066 & 0.0035 & 0.0078 \\
\hline & & 10000 & 6 & 20 & 10 & 23 & 11 & 59 & 30 & 60 & 0.0024 & 0.0112 & 0.0050 & 0.0135 \\
\hline & & 20000 & 6 & 21 & 10 & 23 & 11 & 65 & 30 & 60 & 0.0043 & 0.0192 & 0.0089 & 0.0221 \\
\hline & & 50000 & 6 & 23 & 10 & 23 & 11 & 76 & 30 & 60 & 0.0092 & 0.0488 & 0.0184 & 0.0447 \\
\hline 4 & $x_{0}^{1}$ & 5000 & 1 & 1 & 1 & 1 & 3 & 3 & 3 & 3 & 0.0004 & 0.0004 & 0.0004 & 0.0004 \\
\hline & & 10000 & 1 & 1 & 1 & 1 & 3 & 3 & 3 & 3 & 0.0006 & 0.0006 & 0.0006 & 0.0006 \\
\hline & & 20000 & 1 & 1 & 1 & 1 & 3 & 3 & 3 & 3 & 0.0010 & 0.0009 & 0.0009 & 0.0009 \\
\hline & & 50000 & 1 & 1 & 1 & 1 & 3 & 3 & 3 & 3 & 0.0020 & 0.0021 & 0.0020 & 0.0021 \\
\hline & $x_{0}^{2}$ & 5000 & 4 & 2 & 4 & 6 & 6 & 5 & 6 & 10 & 0.0010 & 0.0007 & 0.0009 & 0.0017 \\
\hline & & 10000 & 4 & 2 & 4 & 6 & 6 & 5 & 6 & 10 & 0.0016 & 0.0011 & 0.0014 & 0.0028 \\
\hline & & 20000 & 4 & 2 & 4 & 6 & 6 & 5 & 6 & 10 & 0.0027 & 0.0018 & 0.0025 & 0.0049 \\
\hline & & 50000 & 4 & 2 & 4 & 8 & 6 & 5 & 6 & 14 & 0.0060 & 0.0039 & 0.0053 & 0.0144 \\
\hline & $x_{0}^{3}$ & 5000 & 5 & 2 & 5 & 10 & 8 & 5 & 8 & 18 & 0.0014 & 0.0008 & 0.0014 & 0.0035 \\
\hline & & 10000 & 5 & 2 & 5 & 10 & 8 & 5 & 8 & 18 & 0.0023 & 0.0011 & 0.0021 & 0.0054 \\
\hline & & 20000 & 5 & 2 & 5 & 10 & 8 & 5 & 8 & 18 & 0.0040 & 0.0020 & 0.0036 & 0.0097 \\
\hline & & 50000 & 5 & 2 & 5 & 10 & 8 & 5 & 8 & 18 & 0.0088 & 0.0041 & 0.0076 & 0.0210 \\
\hline & $x_{0}^{4}$ & 5000 & 7 & 2 & 7 & 14 & 12 & 5 & 12 & 26 & 0.0024 & 0.0008 & 0.0022 & 0.0055 \\
\hline & & 10000 & 7 & 4 & 7 & 14 & 12 & 13 & 12 & 26 & 0.0035 & 0.0031 & 0.0033 & 0.0084 \\
\hline & & 20000 & 7 & 4 & 7 & 15 & 12 & 13 & 12 & 28 & 0.0059 & 0.0052 & 0.0054 & 0.0147 \\
\hline & & 50000 & 7 & 6 & 7 & 15 & 12 & 23 & 12 & 28 & 0.0131 & 0.0201 & 0.0116 & 0.0328 \\
\hline
\end{tabular}


TABLE 2. Numerical results for problem 5-8.

\begin{tabular}{|c|c|c|c|c|c|c|c|c|c|c|c|c|c|c|}
\hline & & & & ITER & & & & $\mathrm{FE}$ & & & & $\mathrm{CPU}$ & & \\
\hline PROB & $x_{0}$ & DIM & NMPCG & PDY & M3TCD1 & sLS & NMPCG & PDY & M3TCD1 & sLS & NMPCG & PDY & M3TCD1 & sLS \\
\hline \multirow[t]{16}{*}{5} & \multirow[t]{4}{*}{$x_{0}^{1}$} & 5000 & 45 & 25 & 49 & 49 & 113 & 95 & 380 & 169 & 0.0492 & 0.0367 & 0.1657 & 0.0736 \\
\hline & & 10000 & 46 & 27 & 48 & 52 & 116 & 104 & 373 & 177 & 0.1119 & 0.0770 & 0.2895 & 0.1493 \\
\hline & & 20000 & 37 & 26 & 49 & 44 & 93 & 101 & 374 & 142 & 0.1508 & 0.1479 & 0.5446 & 0.2289 \\
\hline & & 50000 & 45 & 31 & 45 & 43 & 118 & 126 & 350 & 145 & 0.4376 & 0.5036 & 1.2382 & 0.6233 \\
\hline & \multirow[t]{4}{*}{$x_{0}^{2}$} & 5000 & 51 & 25 & 45 & 52 & 127 & 94 & 350 & 179 & 0.0537 & 0.0601 & 0.1458 & 0.0825 \\
\hline & & 10000 & 45 & 25 & 45 & 43 & 113 & 97 & 351 & 140 & 0.0951 & 0.0797 & 0.3229 & 0.1336 \\
\hline & & 20000 & 50 & 26 & 52 & 46 & 125 & 100 & 408 & 159 & 0.2223 & 0.1982 & 0.7250 & 0.2836 \\
\hline & & 50000 & 49 & 28 & 44 & 43 & 124 & 113 & 344 & 145 & 0.4518 & 0.3983 & 1.1697 & 0.5386 \\
\hline & \multirow{4}{*}{$x_{0}^{3}$} & 5000 & 45 & 23 & 57 & 50 & 113 & 87 & 441 & 172 & 0.0483 & 0.0408 & 0.2055 & 0.0713 \\
\hline & & 10000 & 54 & 23 & 58 & 43 & 124 & 86 & 454 & 140 & 0.1062 & 0.0693 & 0.3843 & 0.1399 \\
\hline & & 20000 & 46 & 28 & 52 & 50 & 116 & 108 & 400 & 171 & 0.2214 & 0.2034 & 0.6746 & 0.2815 \\
\hline & & 50000 & 77 & 28 & 48 & 44 & 173 & 112 & 368 & 142 & 0.7346 & 0.4184 & 1.4102 & 0.5480 \\
\hline & \multirow{4}{*}{$x_{0}^{4}$} & 5000 & 53 & 21 & 58 & 55 & 134 & 77 & 448 & 183 & 0.0564 & 0.0355 & 0.1757 & 0.0761 \\
\hline & & 10000 & 53 & 21 & 60 & 51 & 134 & 77 & 452 & 172 & 0.1029 & 0.0804 & 0.4528 & 0.1433 \\
\hline & & 20000 & 54 & 21 & 78 & 56 & 137 & 77 & 588 & 184 & 0.2121 & 0.1156 & 0.8953 & 0.2990 \\
\hline & & 50000 & 56 & 22 & 54 & 57 & 142 & 80 & 408 & 188 & 0.5549 & 0.3054 & 1.5642 & 0.8342 \\
\hline \multirow[t]{16}{*}{6} & \multirow{4}{*}{$x_{0}^{1}$} & 5000 & 15 & 19 & 5 & 21 & 41 & 71 & 31 & 70 & 0.0107 & 0.0104 & 0.0050 & 0.0107 \\
\hline & & 10000 & 15 & 19 & 5 & 21 & 41 & 71 & 31 & 71 & 0.0115 & 0.0151 & 0.0054 & 0.0172 \\
\hline & & 20000 & 11 & 11 & 5 & 11 & 31 & 40 & 31 & 32 & 0.0155 & 0.0161 & 0.0095 & 0.0152 \\
\hline & & 50000 & 15 & 20 & 5 & 22 & 41 & 76 & 31 & 75 & 0.0451 & 0.0886 & 0.0303 & 0.0735 \\
\hline & \multirow{4}{*}{$x_{0}^{2}$} & 5000 & 15 & 18 & 5 & 19 & 41 & 67 & 32 & 66 & 0.0071 & 0.0095 & 0.0034 & 0.0107 \\
\hline & & 10000 & 15 & 18 & 6 & 19 & 41 & 67 & 40 & 66 & 0.0122 & 0.0151 & 0.0073 & 0.0154 \\
\hline & & 20000 & 15 & 19 & 6 & 20 & 41 & 71 & 40 & 70 & 0.0200 & 0.0368 & 0.0123 & 0.0407 \\
\hline & & 50000 & 15 & 19 & 6 & 20 & 41 & 71 & 40 & 70 & 0.0412 & 0.0557 & 0.0250 & 0.0649 \\
\hline & \multirow[t]{4}{*}{$x_{0}^{3}$} & 5000 & 4 & 5 & 4 & 18 & 7 & 12 & 24 & 64 & 0.0012 & 0.0017 & 0.0026 & 0.0089 \\
\hline & & 10000 & 4 & 5 & 4 & 18 & 7 & 12 & 24 & 64 & 0.0019 & 0.0025 & 0.0036 & 0.0159 \\
\hline & & 20000 & 4 & 5 & 4 & 19 & 7 & 12 & 24 & 68 & 0.0037 & 0.0043 & 0.0070 & 0.0265 \\
\hline & & 50000 & 4 & 5 & 4 & 19 & 7 & 12 & 24 & 68 & 0.0085 & 0.0114 & 0.0155 & 0.0585 \\
\hline & $x_{0}^{4}$ & 5000 & 6 & 19 & 6 & 23 & 11 & 70 & 35 & 77 & 0.0019 & 0.0087 & 0.0035 & 0.0115 \\
\hline & & 10000 & 6 & 21 & 6 & 22 & 11 & 80 & 35 & 70 & 0.0034 & 0.0194 & 0.0064 & 0.0182 \\
\hline & & 20000 & 6 & 21 & 6 & 23 & 11 & 80 & 35 & 74 & 0.0063 & 0.0332 & 0.0111 & 0.0337 \\
\hline & & 50000 & 6 & 21 & 6 & 23 & 11 & 81 & 35 & 72 & 0.0129 & 0.0687 & 0.0219 & 0.0712 \\
\hline 7 & $x_{0}^{1}$ & 5000 & 6 & 16 & 5 & 11 & 11 & 45 & 11 & 23 & 0.0026 & 0.0087 & 0.0022 & 0.0053 \\
\hline & & 10000 & 6 & 16 & 5 & 11 & 11 & 45 & 11 & 24 & 0.0041 & 0.0137 & 0.0035 & 0.0091 \\
\hline & & 20000 & 6 & 16 & 5 & 12 & 11 & 45 & 11 & 27 & 0.0072 & 0.0243 & 0.0061 & 0.0179 \\
\hline & & 50000 & 6 & 17 & 6 & 13 & 11 & 48 & 14 & 30 & 0.0165 & 0.0549 & 0.0174 & 0.0416 \\
\hline & $x_{0}^{2}$ & 5000 & 4 & 14 & 4 & 6 & 6 & 38 & 6 & 10 & 0.0013 & 0.0064 & 0.0012 & 0.0022 \\
\hline & & 10000 & 4 & 14 & 4 & 6 & 6 & 38 & 6 & 10 & 0.0021 & 0.0112 & 0.0020 & 0.0041 \\
\hline & & 20000 & 4 & 15 & 4 & 6 & 6 & 41 & 6 & 10 & 0.0040 & 0.0211 & 0.0038 & 0.0066 \\
\hline & & 50000 & 4 & 15 & 4 & 8 & 6 & 41 & 6 & 14 & 0.0086 & 0.0450 & 0.0077 & 0.0206 \\
\hline & $x_{0}^{3}$ & 5000 & 5 & 17 & 5 & 10 & 8 & 47 & 8 & 18 & 0.0017 & 0.0080 & 0.0016 & 0.0040 \\
\hline & & 10000 & 5 & 17 & 5 & 10 & 8 & 47 & 8 & 18 & 0.0029 & 0.0137 & 0.0028 & 0.0072 \\
\hline & & 20000 & 5 & 18 & 5 & 10 & 8 & 50 & 8 & 18 & 0.0055 & 0.0272 & 0.0052 & 0.0135 \\
\hline & & 50000 & 5 & 18 & 5 & 10 & 8 & 50 & 8 & 18 & 0.0127 & 0.0610 & 0.0106 & 0.0283 \\
\hline & $x_{0}^{4}$ & 5000 & 7 & 19 & 7 & 14 & 12 & 53 & 12 & 26 & 0.0025 & 0.0092 & 0.0023 & 0.0066 \\
\hline & & 10000 & 7 & 19 & 7 & 14 & 12 & 54 & 12 & 26 & 0.0048 & 0.0161 & 0.0044 & 0.0107 \\
\hline & & 20000 & 7 & 20 & 7 & 15 & 12 & 57 & 12 & 28 & 0.0077 & 0.0293 & 0.0071 & 0.0220 \\
\hline & & 50000 & 7 & 21 & 7 & 15 & 12 & 62 & 12 & 28 & 0.0199 & 0.0792 & 0.0183 & 0.0462 \\
\hline 8 & $x_{0}^{1}$ & 5000 & 19 & 15 & 7 & 15 & 54 & 68 & 90 & 61 & 0.0111 & 0.0141 & 0.0172 & 0.0116 \\
\hline & & 10000 & 19 & 15 & 7 & 15 & 54 & 68 & 90 & 61 & 0.0229 & 0.0229 & 0.0283 & 0.0234 \\
\hline & & 20000 & 19 & 16 & 7 & 16 & 54 & 73 & 90 & 65 & 0.0379 & 0.0548 & 0.0713 & 0.0603 \\
\hline & & 50000 & 19 & 17 & 7 & 16 & 54 & 79 & 90 & 65 & 0.0956 & 0.1748 & 0.1509 & 0.1233 \\
\hline & $x_{0}^{2}$ & 5000 & 18 & 14 & 6 & 13 & 51 & 63 & 76 & 46 & 0.0112 & 0.0148 & 0.0122 & 0.0121 \\
\hline & & 10000 & 18 & 15 & 6 & 13 & 51 & 68 & 76 & 46 & 0.0232 & 0.0255 & 0.0240 & 0.0181 \\
\hline & & 20000 & 18 & 15 & 6 & 14 & 51 & 68 & 76 & 51 & 0.0521 & 0.0525 & 0.0563 & 0.0417 \\
\hline & & 50000 & 18 & 16 & 6 & 14 & 51 & 74 & 76 & 51 & 0.1167 & 0.1108 & 0.1315 & 0.1178 \\
\hline & $x_{0}^{3}$ & 5000 & 16 & 13 & 6 & 14 & 45 & 59 & 83 & 58 & 0.0090 & 0.0102 & 0.0130 & 0.0143 \\
\hline & & 10000 & 16 & 13 & 6 & 14 & 45 & 59 & 83 & 58 & 0.0173 & 0.0250 & 0.0389 & 0.0393 \\
\hline & & 20000 & 16 & 13 & 6 & 13 & 45 & 59 & 83 & 47 & 0.0404 & 0.0479 & 0.0834 & 0.0419 \\
\hline & & 50000 & 16 & 14 & 6 & 14 & 45 & 64 & 83 & 52 & 0.0993 & 0.1046 & 0.1674 & 0.1036 \\
\hline & $x_{0}^{4}$ & 5000 & 9 & 15 & 7 & 15 & 21 & 74 & 118 & 59 & 0.0051 & 0.0202 & 0.0411 & 0.0133 \\
\hline & & 10000 & 9 & 15 & 7 & 15 & 21 & 74 & 118 & 59 & 0.0198 & 0.0249 & 0.0473 & 0.0307 \\
\hline & & 20000 & 9 & 14 & 7 & 16 & 21 & 70 & 118 & 64 & 0.0191 & 0.0672 & 0.1010 & 0.0690 \\
\hline & & 50000 & 9 & 19 & 7 & 16 & 21 & 104 & 118 & 64 & 0.0409 & 0.1871 & 0.1871 & 0.1413 \\
\hline
\end{tabular}




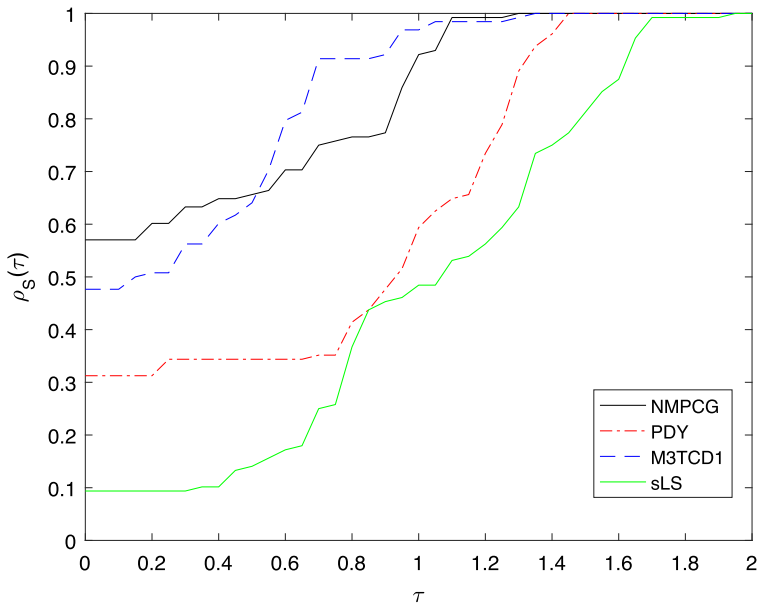

FIGURE 1. Iterations performance profile.

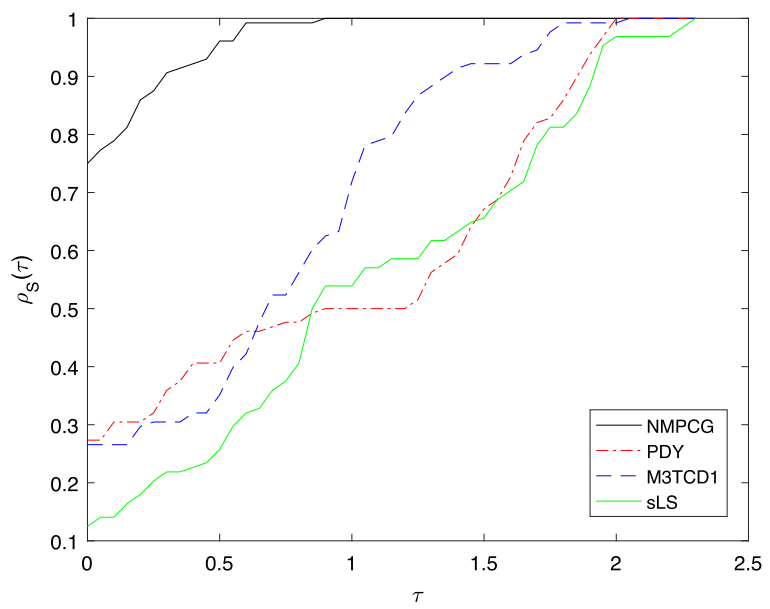

FiguRE 2. Function evaluations performance profile.

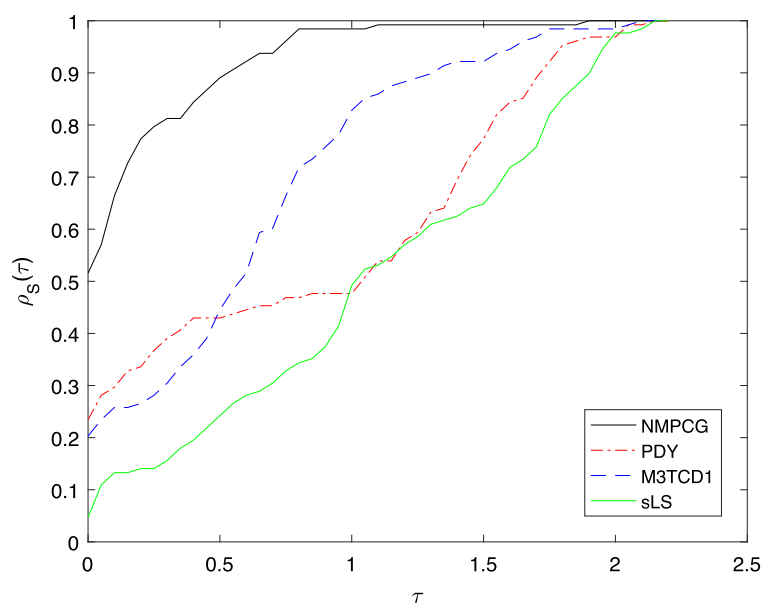

FiguRE 3. CPU time performance profile. 
For a comprehensive performance of the methods, we use the performance profiles tool proposed by Dolan and Moré [10] to represent the results in Tables 1 and 2 in figures, based on the number of iterations, number of function evaluations, and CPU time. These are presented in Figures 1-3, respectively. With the Dolan and Moré performance profiles, the graph that is above the others for the most part is regarded as the best solver. We can see from Figure 1 that in terms of number of iterations, the NMPCG and M3TCD1 methods are very competitive, and perform much better than both the $P D Y$ and $s L S$ methods. Figure 2 shows clearly that in terms of function evaluations, the proposed $N M P C G$ method outperforms all the other methods, followed by the $M 3 T C D 1$ method. Figure 3, for the $C P U$ performance profiles, shows that the proposed $N M P C G$ is equally efficient.

\section{Conclusion}

In this paper, we proposed a new modified derivative-free Perry's conjugate gradient-based projection method for solving systems of large-scale nonlinear monotone equations. Its global convergence and rate of convergence were established. The proposed algorithm was tested on some benchmark problems, with different starting points and dimensions, and the numerical results show that the method is efficient as compared to other methods from the literature. Future work includes extending the new method to solve other kinds of problems like robotic motion control, signal restoration and image deblurring.

Acknowledgements. The authors are grateful to the Editor and the anonymous referees for their valuable suggestions and comments that improved the quality of this work.

\section{REFERENCES}

[1] A.B. Abubakar and P. Kumam, A descent Dai-Liao conjugate gradient method for nonlinear equations. Numer. Algor. 81 (2019) 197-210.

[2] A.B. Abubakar, J. Rilwan, S.E. Yimer, A.H. Ibrahim and I. Ahmed, Spectral three-term conjugate descent method for solving nonlinear monotone equations with convex constraints. Thai J. Math. 18 (2020) 501-517.

[3] N. Andrei, Scaled conjugate gradient algorithms for unconstrained optimization. Comput. Optim. Appl. 38 (2007) $401-416$.

[4] A.M. Awwal, P. Kumam, H. Mohammad, W. Watthayu and A.B. Abubakar, A Perry-type derivative-free algorithm for solving nonlinear system of equations and minimizing $l_{1}$ regularized problem. Optimization $\mathbf{7 0}$ (2021) 1231-1259.

[5] Y.H. Dai and L.Z. Liao, New conjugacy conditons and related nonlinear conjugate gradient methods. Appl. Math. Optim. 43 (2001) $87-101$.

[6] Y.H. Dai, Y.K. Huang and X.W. Liu, A family of spectral gradient methods for optimization. Comput. Optim. Appl. 74 (2019) $43-65$.

[7] Z. Dai, X. Chen and F. Wen, A modified Perry's conjugate gradient method-based derivative-free method for solving large-scale nonlinear monotone equations. Appl. Math. Comput. 270 (2015) 378-386.

[8] Y. Ding, Y. Xiao and J. Li, A class of conjugate gradient methods for convex constrained monotone equations. Optimization 66 (2017) 2309-2328.

[9] S.P. Dirkse and M.C. Ferris, A collection of nonlinear mixed complementarity problems. Optim. Math. Softw. 5 (1995) 319-345.

[10] E.D. Dolan and J.J. More, Benchmarking optimization software with performance profiles. Math. Program. 91 (2002) $201-213$.

[11] P. Gao, C. He and Y. Liu, An adaptive family of projection methods for constrained monotone equations with applications. Appl. Math. Comput. 359 (2019) 1-16.

[12] A.S. Halilu, A. Majumder, M.Y. Waziri and K. Ahmed, Signal recovery with convex constrained nonlinear monotone equations through conjugate gradient hybrid approach. Math. Comput. Simulat. 187 (2021) 520-539.

[13] P. Kaelo and M. Koorapetse, A global convergent projection method for systems of nonlinear monotone equations. Int. J. Comput. Math. 98 (2021) 421-434.

[14] M. Koorapetse, P. Kaelo and E.R. Offen, A scaled derivative-free projection method for solving nonlinear monotone equations. Bull. Iran. Math. Soc. 45 (2019) 755-770.

[15] M. Koorapetse, P. Kaelo, S. Lekoko and T. Diphofu, A derivative-free RMIL conjugate gradient projection method for convex constrained nonlinear monotone equations with applications in compressive sensing. Appl. Numer. Math. 165 (2021) $431-441$.

[16] J. Liu and Y. Feng, A derivative-free iterative method for nonlinear monotone equations with convex constraints. Numer. Algor. 82 (2019) 245-262.

[17] P. Liu, J. Jian and X. Jiang, A new conjugate gradient projection method for convex constrained nonlinear equations. Complexity 2020 (2020) 1-14. 
[18] J.K. Liu, J.L. Xu and L.Q. Zhang, Partially symmetrical derivative-free Liu Storey projection method for convex constrained equations. Int. J. Comput. Math. 96 (2019) 1787-1798.

[19] I.E. Livieris and P. Pintelas, Globally convergent modified Perry's conjugate gradient method. Appl. Math. Comput. 218 (2012) 9197-9207.

[20] K. Meintjes and A.P. Morgan, A methodology for solving chemical equilibrium systems. Appl. Math. Comput. 22 (1987) $333-361$.

[21] Y. Ou and J. Li, A new derivative-free SCG-type projection method for nonlinear monotone equations with convex constraints. J. Appl. Math. Comput. 56 (2018) 195-216.

[22] A. Perry, A modified conjugate gradient algorithm. Oper. Res. 26 (1978) 1073-1078.

[23] J. Sabi'u, A. Shah and M.Y. Waziri, Two optimal Hager-Zhang conjugate gradient methods for solving monotone nonlinear equations. Appl. Numer. Math. 153 (2020) 217-233.

[24] D. Shanno, On the convergence of a new conjugate gradient algorithm. SIAM J. Numer. Anal. 15 (1978) $1247-1257$.

[25] M.V. Solodov and B.F. Svaiter, A Globally Convergent Inexact Newton Method for Systems of Monotone Equations, edited by M. Fukushima, L. Qi. In: Reformulation: Nonsmooth, Piecewise Smooth, Semismooth and Smoothing Methods, Vol. 22 of Applied Optimization. Springer, Boston, MA (1998), 355-369.

[26] M. Sun and J. Liu, New hybrid conjugate gradient projection method for the convex constrained equations. Calcolo 53 (2016) 399-411.

[27] M.Y. Waziri, K. Ahmed and J. Sabi'u, A Dai-Liao conjugate gradient method via modified secant equation for system of nonlinear equations. Arab. J. Math. 9 (2020) 443-457.

[28] M.Y. Waziri, K. Ahmed, J. Sabi'u and A.S. Halilu, Enhanced Dai-Liao conjugate gradient methods for systems of nonlinear equations. SeMA J. $\mathbf{7 8}$ (2021) 15-51.

[29] Z. Wei, G. Li and L. Qi, New quasi-Newton methods for unconstrained optimization problems. Appl. Math. Comput. 175 (2006) 1156-1188.

[30] S. Yao and L. Ning, An adaptive three-term conjugate gradient method based on self-scaling memoryless BFGS matrix. J. Comput. Appl. Math. 332 (2018) 72-85.

[31] Q.-R. Yan, X.-Z. Peng and D.-H. Li, A globally convergent derivative-free method for solving large-scale nonlinear monotone equations. J. Comput. Appl. Math. 234 (2010) 649-657.

[32] L. Zhang, W.J. Zhou and D.H. Li, A descent modified Polak-Ribiére-Polyak conjugate gradient method and its global convergence. IMA J. Numer. Anal. 26 (2006) 629-640.

[33] L. Zheng, L. Yang and Y. Liang, A modified spectral gradient projection method for solving non-linear monotone equations with convex constraints and its application. IEEE Acess 99 (2020) 1-1.

[34] W.Y. Zhao and D. Li, Monotonicity of fixed point and normal mapping associated with varriational inequality and its application. SIAM. J. Optim. 11 (2000) 962-973.

[35] L. Zheng, L. Yang and Y. Liang, A conjugate gradient projection method for solving equations with convex constraints. J. Comput. Appl. Math. 375 (2020) 399-411.

\section{Subscribe to Open (S20) A fair and sustainable open access model}

This journal is currently published in open access under a Subscribe-to-Open model (S2O). S2O is a transformative model that aims to move subscription journals to open access. Open access is the free, immediate, online availability of research articles combined with the rights to use these articles fully in the digital environment. We are thankful to our subscribers and sponsors for making it possible to publish this journal in open access, free of charge for authors.

\section{Please help to maintain this journal in open access!}

Check that your library subscribes to the journal, or make a personal donation to the $\mathrm{S} 2 \mathrm{O}$ programme, by contacting subscribers@edpsciences.org

More information, including a list of sponsors and a financial transparency report, available at: https://www. edpsciences.org/en/maths-s2o-programme 